\title{
zons

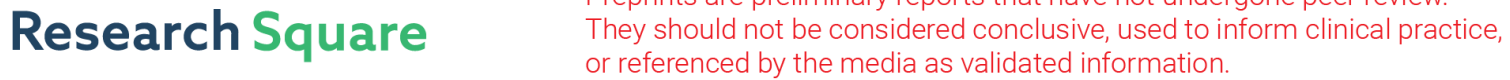 \\ Evaluation of Small Intestinal Motility in a Rat Model of Irritable Bowel Syndrome
}

\section{Masamichi Sato}

Juntendo University Graduate School of Medicine

\section{Takahiro Kudo ( $\nabla$ t-kudo@juntendo.ac.jp )}

Juntendo University Graduate School of Medicine

\section{Nobuyasu Arai}

Juntendo University Graduate School of Medicine

\section{Reiko Kyodo}

Juntendo University Graduate School of Medicine

\section{Kenji Hosoi}

Juntendo University Graduate School of Medicine

\section{Keita Sakaguchi}

Juntendo University Graduate School of Medicine

\section{Tamaki lkuse}

Juntendo University Graduate School of Medicine

\section{Keisuke Jimbo}

Juntendo University Graduate School of Medicine

\section{Yoshikazu Ohtsuka}

Juntendo University Graduate School of Medicine

\section{Toshiaki Shimizu}

Juntendo University Graduate School of Medicine

\section{Research Article}

Keywords: enterochromaffin cells, gastrointestinal motility, irritable bowel syndrome, serotonin, serotonin 3 receptor

Posted Date: August 5th, 2021

DOI: https://doi.org/10.21203/rs.3.rs-763246/v1

License: (9) (1) This work is licensed under a Creative Commons Attribution 4.0 International License. Read Full License 


\section{Abstract}

Background: The correlation between small intestinal motility alteration and irritable bowel syndrome (IBS) is not well evaluated.

Aims: To assess the small intestinal and colonic transits in an IBS rat model with restraint stress and determine the role of small intestinal motility in the IBS pathophysiology.

Methods: Restraint stress was utilized to make adolescent IBS rat models that were evaluated for clinical symptoms, including stool frequency and diarrhea. The small intestinal motility and transit rate were also evaluated. The amounts of mRNA encoding corticotropin-releasing hormone, mast cell, and serotonin (5Hydroxytryptamine; 5 -HT) receptor $3 a$ were quantified using real-time polymerase chain reaction (PCR); the 5-HT expression was evaluated using immunostaining.

Results: Restraint stress significantly increased the number of fecal pellet outputs, stool water content, and small intestinal motility in the IBS rat models. There was no difference in real-time PCR results, but immunostaining analysis revealed that $5-\mathrm{HT}$ expression in the small intestine was significantly increased in the IBS rat models.

Conclusions: In the adolescent rat model of IBS with restraint stress, we observed an increase in small intestinal and colonic motility. In the small intestine, enhanced 5-HT secretion in the distal portion may be involved in increasing the small intestinal motility.

\section{Introduction}

Irritable bowel syndrome (IBS) is a common functional gastrointestinal disorder characterized by the symptoms of abdominal pain and altered bowel habits, such as diarrhea or constipation, in the absence of any organic diseases. Although the pathophysiology of this disease remains uncertain, sufficient compelling evidence has accumulated to indicate that IBS is a multifactorial syndrome resulting from interactions among gastrointestinal motility, visceral hypersensitivity, intestinal inflammation, gastrointestinal infection, fecal flora alterations, bacterial overgrowth, food sensitivity, genetic factors, and psychosocial dysfunction [1].

Colonic transit disorders may contribute to symptoms among patients with IBS; in fact, transit and contractile abnormalities have been observed in a subset of patients with IBS. The primary alteration of mucosal absorption and secretion has been suggested as non-contributory to the frequent stools in IBS patients with diarrhea [2].

Several previous studies have shown a relationship between the accelerated transit of colonic contents with IBS and diarrhea. Mönnikes et al. reported a significant acceleration of colonic transit in rats with restraint stress compared to non-restrained rats [3]. 
Furthermore, visceral sensation hypersensitivity from the autonomic nervous system that is associated with the brain-gut interaction is thought to be a pathophysiology of IBS [4]. Corticotropin-releasing hormone $(\mathrm{CRH})$ is an important factor in explaining the pathophysiology of brain-gut interactions. $\mathrm{CRH}$ stimulates pituitary adrenocorticotropic hormone and increases intestinal motility in the human body [5]. Recently, there has been a growing interest in serotonin (5-Hydroxytryptamine; 5-HT) due to its possible involvement in IBS. 5-HT released by enterochromaffin cells (EC cells) within the mucosa via intraluminal distension or irritation stimulates 5 -HT3 receptors located on the primary afferent neurons of both splanchnic and vagal fibers, thereby modulating a sensory response [6].

Although increased stool water in diarrhea is thought to result from diminished contact time of the luminal contents with the colonic mucosa, it is well known that approximately $80 \%$ of ingested water is absorbed from the small intestinal mucosa. Although it is clear that small intestine dysfunction induces diarrhea in many diseases, the correlation between diarrhea and motility alteration in the small intestine among patients with IBS has not been well evaluated. In this study, we aimed to assess the small intestinal transit in adolescent IBS rat models with restraint stress and to determine the role of small intestinal motility in the pathophysiology of IBS.

\section{Materials And Methods}

\section{Animal models}

The experiments were performed using adolescent male Sprague-Dawley rats aged 5-6 weeks (to avoid female hormonal effects) weighing 160-250 g (CLEA Japan, Inc., Tokyo), which were housed in cages in a standardized environment with temperature of $24{ }^{\circ} \mathrm{C}$, relative humidity of $55 \% \pm 15 \%$, and a 12-hour/12hour light-dark cycle for a day. They were allowed to access food and water freely. The animal care and experimental protocols were approved by the Institutional Review Board of Juntendo University.

The animals were randomly assigned to two groups: a restraint group and a control group. The restraint group was placed in isolation among individual compartments of stress cages (Natsume Seisakusho Co. Ltd. Tokyo, Japan; KN-325-C-3) for 1 hour before dissection. Rats in the control group assumed an hour of isolation in the cages without restriction. The rats in both groups did not have free access to food and water during isolation.

All methods were carried out in accordance with relevant guidelines and regulations and this study was carried out in compliance with the ARRIVE guidelines as rats were used in this study.

\section{Fecal pellet output and water contents}

The number of fecal pellet outputs during the one-hour isolation was counted. The stool first excreted during isolation was collected, as well as the stool located in the most distal side of their gastrointestinal tract after isolation, for comparison. These fecal pellets were stored at a temperature of $-80{ }^{\circ} \mathrm{C}$ and were freeze-dried overnight after weighing. The next day, the freeze-dried fecal weight was measured to 
calculate the water content (\%), which was as follows: (fecal weight before drying - fecal weight after drying) / fecal weight before drying $\times 100 \%$.

\section{Small intestinal transit}

We examined the intestinal propulsion of powdered carbon to evaluate small intestinal motility under restraint stress. The rats received $0.5 \mathrm{~mL}$ of a powdered carbon suspension in saline per $100 \mathrm{~g}$ of their weight (5\% W/V) intragastrically through an oral sonde (Primetech Co. Ltd. Tokyo, Japan; FTP-15-78-50). After administration, the restraint group rats were immediately exposed to stress as described above. Rats in both groups underwent an autopsy at 1 hour after intragastric administration of the powdered carbon suspension. The small intestines were collected and their entire lengths were measured. We also measured the length of the small intestine containing the carbon marker. The small intestinal transit rate (\%) was calculated as follows: (the length of the small intestine containing the marker / total small intestinal length) $\times 100 \%$.

\section{Real-time PCR}

The amount of mRNA encoding $\mathrm{CRH}$, mast cells, and 5-HTR3a were quantified using real-time PCR. Fullthickness segments of the small intestine and proximal and distal colonic tissue samples were preserved in RNA stabilization solution and stored at $-80^{\circ} \mathrm{C}$ until use. Each tissue sample was homogenized in Tri Reagents (Tomy, Japan; MS100) for the extraction of total RNA according to the manufacturer's instructions (Applied Biosystems). Real-time PCR was performed using the 7500 Fast Real-Time PCR system (Applied Biosystems). The expression of each gene was normalized to the expression of GAPDH using the standard curve method. TaqMan was used for analysis using primers for CRH (Assay number Rn01462137_m1), mast cells (Assay number Rn04342812_g1), and 5-HTR3a (Assay number Rn00667026_m1). The results were compared between the restraint and control groups for both the small intestine and colon. Furthermore, the proximal and distal segments of the small intestine were also compared.

\section{Immunohistochemical analysis}

Small intestine and colon tissues were dissected from rats and fixed in 4\% paraformaldehyde in 100-mM phosphate buffer at room temperature for 24 hours. Serial sections (4- $\mu \mathrm{m}$ thick) were prepared from formalin-fixed paraffin-embedded tissue sections of the small intestine and colon. For 5-HT detection, cells were incubated with 5HT (1: 10; Thermo Fisher Scientific, Rockford, USA), and then stained using the IVIEW $^{\mathrm{TM}}$ DAB Detection Kit (Ventana) and Hematoxylin Counterstain II (Ventana). For 5HTR3a detection, paraffin sections were heat-treated in Cell Conditioning Solution (CC1) (Ventana Medical Systems) for 5-HTR3a, incubated with normal horse serum (Vector), rabbit anti-rat 5-HTR3a (Abcam), HRP-polymer-conjugated horse anti-rabbit IgG (Vector), and then stained using ultraView ${ }^{\text {TM }}$ Universal DAB Detection Kit (Ventana) and Hematoxylin Counterstain II (Ventana). All the stains below were used according to the manufacturer's protocol. An automated immunostainer (BenchMark; Ventana) was used to stain both 5-HT and 5-HTR3a. For each specimen, the number of 5-HT positive cells was 
counted in six randomly selected fields per section using a KS400 Image Analyzer System (Zeiss). The data were expressed as the average number of positive cells per $400 \mu \mathrm{m}^{2}$ of the mucosa.

Similar to real-time PCR, the restraint and control groups were compared for both the small intestine and colon, and distal and proximal comparisons were also made in the small intestine.

\section{Statistical methods}

All experimental data are presented as the mean \pm standard deviation. All statistical analyses were performed using EZR [18] (Saitama Medical Center, Jichi Medical University, Saitama, Japan). More precisely, EZR is a modified version of $\mathrm{R}$ commander (version 2.7-0, 2021) designed to add statistical functions frequently used in biostatistics. As appropriate, the $t$-test was used for comparisons between the two groups. Differences between means at a level of $p<0.05$ were defined as statistically significant.

\section{Data Accessibility}

The data that support the findings of this study are available from Juntendo University, but restrictions apply to the availability of these data, as they were used under license for the current study and are not publicly available. However, data are available from the authors upon reasonable request and with the permission of Juntendo University.

\section{Results}

\section{Fecal pellet output and water content}

We counted the fecal pellet outputs of 12 restraint rats and 12 control rats. The mean number of fecal pellets was $6.9 \pm 1.7$ and $0.8 \pm 0.8$ in the restraint and control groups, respectively, showing that restraint stress significantly increased the number of fecal pellet outputs $(p<0.001$, Fig. $1 \mathrm{~A})$. We also calculated the water content of fecal pellets in six restraint rats and six control rats. The mean water content of the first stool during isolation was $75.5 \% \pm 7.5 \%$ and $74.7 \% \pm 5.4 \%$ in the restraint and control groups, respectively, with no significant difference in primary stools between the two groups during isolation ( $p=$ $0.80)$. On the other hand, the mean water content of the fecal pellets after isolation was significantly different between the restraint rats and control rats, at $94.8 \% \pm 4.2 \%$ and $79.1 \% \pm 6.0 \%$, respectively $(p<$ 0.001 , Fig. 1B). This result indicates that restraint stress led to increased water content in the stool and induced diarrhea in the stress models.

\section{Small intestinal transit}

Nine restraint rats and 16 control rats were compared for small intestinal motility. The average length of the small intestine was not significantly different between the restraint and control groups $(115.3 \pm 3.8$ cm vs $113.3 \pm 9.2 \mathrm{~cm} ; p=0.63$ ) (Fig. $1 \mathrm{C}$ ). The average small intestinal transit rate was $77.9 \% \pm 7.4 \%$ in the 
restraint group and $69.5 \% \pm 8.9 \%$ in the control group, with the restraint rats showing a significant increase in the small intestinal transit rate $(p<0.03)$ (Fig. 1D).

\section{Real-time polymerase chain reaction (PCR)}

Six restraint rats and six control rats were compared for the expression of $\mathrm{CRH}$, mast cells, and 5Hydroxytryptamine Receptor 3A (5-HTR3a) in the small intestine and colon using real-time PCR. The transcripts encoding these were normalized to those encoding glyceraldehyde 3-phosphate dehydrogenase (GAPDH). We found that the mRNA expression was not significantly different between the restraint and control rats (Table 1). Examining the segment of the small intestine, 5-HTR3a expression was significantly increased at the distal portion compared to the proximal section in both the restraint and control groups (restraint: $p=0.009$; control: $p=0.01$ ) (Fig. $2 \mathrm{~A}, \mathrm{~B}$ ). Moreover, in the distal small intestine, there was no significant difference in 5-HTR3a expression between the restraint and control groups (0.06 \pm 0.03 vs. $0.04 \pm 0.01: p=0.16)$ (Fig. $2 \mathrm{C})$.

Table 1

Amount of mRNA encoding $\mathrm{CRH}$, mast cell, and 5-HT receptor 3a mRNA expression in the small colon and colon as quantified by real-time PCR

\begin{tabular}{|lllll|}
\hline & & CRH & Mast cell & 5-HTR3a \\
\hline Small intestine & Restraint group & - & $1.50 \pm 1.50$ & $0.03 \pm 0.02$ \\
\hline & Control group & - & $2.36 \pm 2.42$ & $0.04 \pm 0.03$ \\
& P value & - & 0.408 & 0.336 \\
\hline & Restraint group & - & $1.28 \pm 1.00$ & $1.94 \pm 0.93$ \\
\hline Colon & Control group & - & $1.37 \pm 0.96$ & $1.25 \pm 0.68$ \\
\hline & P value & - & 0.81 & 0.05 \\
\hline $\begin{array}{l}\text { Values are mean } \pm \text { standard deviation [SD]. There was no difference in CRH, 5-HT receptor and mast } \\
\text { cell subunits between the restraint and control groups. }\end{array}$ \\
\hline
\end{tabular}

\section{Immunohistochemical analysis}

Immunostaining of 5-HT and 5-HTR3a was performed using tissues from the small intestine and colon. Six restraint rats and six control rats were used for comparison. Since 5-HT is secreted by EC cells, the 5HT antibody staining of the small intestine and colon tissues was used to stain the EC cells. Positive cells in the control and restraint groups were counted and compared. As a result, EC cell expression tended to increase in the restraint group compared to the control group in both the small intestine and colon (Fig. 3A, B). The number of positive cells was significantly higher in the restraint group than in the control group in the small intestine instead of the colon (small intestine: $p=0.004$; colon: $p=0.3$ ) (Fig. 4). When the number of EC cells in the small intestine was compared between the proximal and distal small intestine, no significant difference was observed between the restraint and control groups (restraint 
group: $p=0.95$; control group: $p=0.48$ ) (Fig. $5 \mathrm{~A}, \mathrm{~B})$. In the distal small intestine, the number of EC cells significantly increased in the restraint group compared to the control group ( $1.33 \pm 0.52$ vs. $0.53 \pm 0.18$, respectively; $p=0.012$ ) (Fig. $6 \mathrm{~A})$. In the proximal small intestine, there was no significant difference in the number of EC cells between the restraint and control groups ( $1.36 \pm 0.82$ vs. $0.67 \pm 0.37$, respectively; $p=$ 0.13) (Fig. 6B). Moreover, there was no significant difference in 5-HTR3a between the control and restraint groups in both the small intestine and colon (Fig. 7).

\section{Discussion}

Animal models of bowel motility dysfunction associated with stress induced by cold, acoustics, ether, cold restraint, and wrap restraint have been previously reported. For example, Murakami et al. [7] intravenously administered $\mathrm{CRH}$ to induce stress in rats. On the other hand, Bradesi et al. [8] used wateravoidance stress. Furthermore, restraint stress has also been used in rat stress models. The number of defecations in rats reportedly increase by restraining the body [9]. In the present study, rats subjected to restraint alone at room temperature showed an increase in fecal pellet output without the formation of gastrointestinal lesions. We chose restraint stress to make IBS rat models, because this stress method is simple and provides equivalent stress for each rat. In humans, IBS is diagnosed based on the clinical symptoms. Even in the animal models created in our experiment, symptoms are very important to confirm IBS. In the present study, rats showed an increased number of fecal pellet output and diarrhea as clinical symptoms. Although we could not evaluate visceral hypersensitivity as no increased $\mathrm{CRH}$ release was observed, it was possible to create IBS rat models.

IBS was previously called irritable colon syndrome [10]. The reason the "colon" was changed to "bowel" is because IBS causes abnormal movement in the entire digestive tract. Currently, most studies on IBS focus on visceral hypersensitivity and the brain-gut interaction. Therefore, it is important to understand gastrointestinal motility during IBS treatment. There are some reports about colon motility in IBS. For example, Nakade et al. [11] reported that rats with restraint stress had significantly accelerated colonic transit compared to non-restrained rats. Kellow et al. [12] reported that the increase in small intestinal motility was greater in diarrhea-type IBS than in constipation-type IBS. On the other hand, Hebden et al. [13] reported that the small intestinal transit time of patients with diarrhea-type IBS was shorter than that of healthy people. However, Hardy et al. [14] reported that when comparisons were made between diarrhea-type and constipation-type IBS, it was observed that the colonic transit time in diarrhea-type IBS was shorter, whereas no difference in transit time was observed in the small intestine. Therefore, there was no influence on the small intestine movement in IBS. In the present study, however, small intestinal motility was significantly elevated, as observed in the small intestinal transit rate. Furthermore, colon motility was increased, as observed in the fecal pellet output and water content examination. Our IBS rat model had a shortened transit time in the entire intestine.

In this study, 5-HT, 5-HTR3a, and mast cells were evaluated in the colon and small intestine as factors that regulate the movement of the intestinal tract. Most 5-HT is synthesized and stored in EC cells, and 5$\mathrm{HT}$ receptors are located throughout the intestinal tract. There are seven families of 5-HT receptors (5- 
HTR1 to 5-HTR7). In the gastrointestinal tract, 5-HTR3 and 5-HTR4 subtypes have been the most studied. In particular, 5-HTR3 antagonists have been shown to reduce visceral hypersensitivity and pain in IBS patients [15]. Therefore, 5-HT and 5-HTR3 play important roles in the gastrointestinal tract motility. However, their expression in the small intestine remains unclear. In our study, the motility of the small intestine in the restraint group was significantly enhanced. Therefore, an increase in 5-HT and 5-HTR3 levels in the small intestine was expected. In the restraint group, EC cell expression in the small intestine was significantly increased, but not 5-HTR3a. This result suggests that 5-HT has a strong effect on the enhancement of small intestinal motility under acute stress. When the increase in EC cell expression in the small intestine among rats in the restraint group was evaluated with respect to the segment of the small intestine, we observed that the expression was significantly increased in the distal small intestine as compared to that in the proximal small intestine. Furthermore, real-time PCR did not show a stressrelated increase in 5-HTR3a in the small intestine, but the distal small intestine expressed more 5-HTR3a than the proximal small intestine in both the restraint and control groups. Therefore, the distal small intestine may be mainly involved in small intestinal motility. The high expression of EC cells in the small intestine may result in increased intestinal motility and may be involved in the development of diarrheal symptoms in IBS. This suggests that the small intestine may be more susceptible to stress than the colon. In our study, real-time PCR showed no significant difference in mast cells between the restraint and control groups. However, there are some studies on increased mast cells in patients with IBS. Chadwick et al. [16] reported that the number of mast cells increased in the colonic mucosa of patients with IBS. Weston et al. [17] reported that the number of these cells increased in the ileal mucosa of patients with IBS. It is possible that the reason the mast cells did not increase in our study was because the stress introduced was acute and not chronic. However, it remains unclear whether stress and IBS are correlated with an increase in mast cells. Mast cells are also known to be increased by inflammation. Based on these facts, it can be said that the IBS rat model created in our study had no inflammation and that the IBS rat model could be accurately created.

Nevertheless, our study had some limitations. First, in the creation of IBS model rats, the stress was acute, not chronic. While it might have been better if we chose chronic stress because IBS is a chronic syndrome, there was the possibility of ulcer formation due to chronic stress, which we wanted to avoid. We decided that chronic stress causing intestinal ulceration should not be a part of the evaluation, since this study dealt with functional intestinal disorders. Second, we were unable to evaluate increased CRH release. We tried to evaluate the increase in CRH using RT-PCR, but it was not detected. Therefore, we determined whether the IBS rat model could be created based on clinical symptoms. Third, 5-HTR3a was evaluated using immunostaining, but the difference in expression levels could not be quantified. RT-PCR did not show a significant increase in 5-HTR3a expression in both the small intestine and colon. If the expression level had been quantified using immunostaining, the evaluation would be more accurate. In this study, the evaluation focused on $5-\mathrm{HT}$. However, there are other factors known to be involved in intestinal motility. We would like to evaluate these factors in future studies.

\section{Conclusion}


IBS model rats could be created by applying restraint stress. We found that the small intestine was prominently involved in the enhancement of intestinal peristalsis, which causes diarrhea. In particular, the distal small intestine may be significantly involved in the enhancement of small intestine movement due to acute stress loading.

\section{Declarations}

\section{Funding}

None.

\section{Competing interest}

The authors declare no competing interests.

\section{Author contributions}

Formulation of ideas, research goals and aims, development or design of methodology: TK, MS, TI, and KS

Data curation and formal analysis: MS, TI, TK, and YO

Conducting the research and investigation process, specifically performing the experiments, or data/evidence collection: NA, RK, KH, and KJ

Oversight and leadership responsibility for the research activity planning and execution, including mentorship external to the core team: TS

Creation and/or presentation of the published work, specifically writing the initial draft: MS, TK, and YO Critical review, commentary, or revision: TI and TK

\section{Acknowledgments}

The authors thank Ms. Yumiko Sakurai from the Department of Pediatric and Adolescent Medicine, Juntendo University Graduate School of Medicine and the members of the Laboratory of Morphology and Image Analysis, Research Support Center, Juntendo University Graduate School of Medicine for providing technical assistance with microscopy. We would like to thank Editage (www.editage.com) for English language editing.

\section{References}

1. Camilleri, M., Lasch, K. \& Zhou, W. Irritable bowel syndrome: methods, mechanisms, and pathophysiology. The confluence of increased permeability, inflammation, and pain in irritable bowel 
syndrome. Am. J. Physiol. Gastrointest. Liver Physiol.303, G775-G785 (2012).

2. Bazzocchi, G., et al. Effect of eating on colonic motility and transit in patients with functional diarrhea. Simultaneous scintigraphic and manometric evaluations. Gastroenterology101, 1298-1306 (1991).

3. Mönnikes, H., Schmidt, B. G., Raybould, H. E. \& Taché, Y. CRF in the paraventricular nucleus mediates gastric and colonic motor response to restraint stress. Am. J. Physiol.262, G137-G143 (1992).

4. Drossman, D. A. The functional gastrointestinal disorders and the Rome III process. Gastroenterology130, 1377-1390 (2006).

5. Fukudo, S., Nomura, T. \& Hongo, M. Impact of corticotropin-releasing hormone on gastrointestinal motility and adrenocorticotropic hormone in normal controls and patients with irritable bowel syndrome. Gut42, 845-849 (1998).

6. Atkinson, W., Lockhart, S., Whorwell, P. J., Keevil, B. \& Houghton, L. A. Altered 5-hydroxytryptamine signaling in patients with constipation- and diarrhea-predominant irritable bowel syndrome. Gastroenterology130, 34-43 (2006).

7. Murakami, T. et al. Changes in intestinal motility and gut microbiota composition in a rat stress model. Digestion95, 55-60 (2017).

8. Bradesi, S. et al. Repeated exposure to water avoidance stress in rats: a new model for sustained visceral hyperalgesia. Am. J. Physiol. Gastrointest. Liver Physiol.289, G42-G53 (2005).

9. Miyata, K. et al. Role of the serotonin3 receptor in stress-induced defecation. J. Pharmacol. Exp. Ther.261, 297-303 (1992).

10. Connell, A. M. The irritable colon syndrome. Postgrad. Med. J.44, 668-674 (1968).

11. Nakade, Y. et al. Restraint stress stimulates colonic motility via central corticotropin-releasing factor and peripheral 5-HT3 receptors in conscious rats. Am. J. Physiol. Gastrointest. Liver Physiol.292, G1037-G1044 (2007).

12. Kellow, J. E. \& Phillips, S. F. Altered small bowel motility in irritable bowel syndrome is correlated with symptoms. Gastroenterology 92 , 1885-1893 (1987).

13. Hebden, J. M., Blackshaw, E., D'Amato, M., Perkins, A. C. \& Spiller, R. C. Abnormalities of GI transit in bloated irritable bowel syndrome: effect of bran on transit and symptoms. Am. J. Gastroenterol.97, 2315-2320 (2002).

14. Hardy, J. G., Wood, E., Clark, A. G. \& Reynolds, J. R. Whole-bowel transit in patients with the irritable bowel syndrome. Eur. J. Nucl. Med.11, 393-396 (1986).

15. Bardhan, K. D. et al. A double-blind, randomized, placebo-controlled dose-ranging study to evaluate the efficacy of alosetron in the treatment of irritable bowel syndrome. Aliment. Pharmacol. Ther.14, 23-34 (2000).

16. Chadwick, V. S. et al. Activation of the mucosal immune system in irritable bowel syndrome. Gastroenterology122, 1778-1783 (2002). 
17. Weston, A. P., Biddle, W. L., Bhatia, P. S. \& Miner, P. B. Jr. Terminal ileal mucosal mast cells in irritable bowel syndrome. Dig. Dis. Sci.38, 1590-1595 (1993).

18. Kanda, Y. Investigation of the freely available easy-to-use software 'EZR' for medical statistics. Bone Marrow Transplant.48, 452-458 (2013).

\section{Figures}

A)

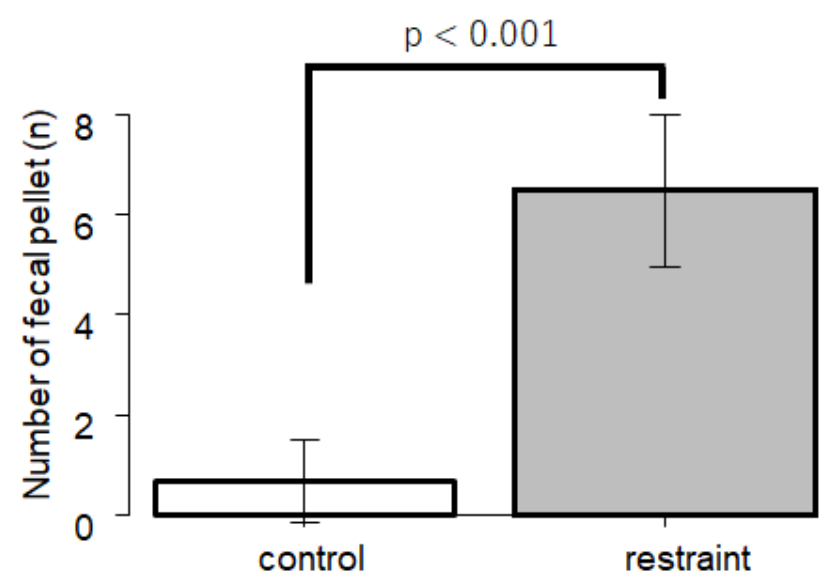

C)

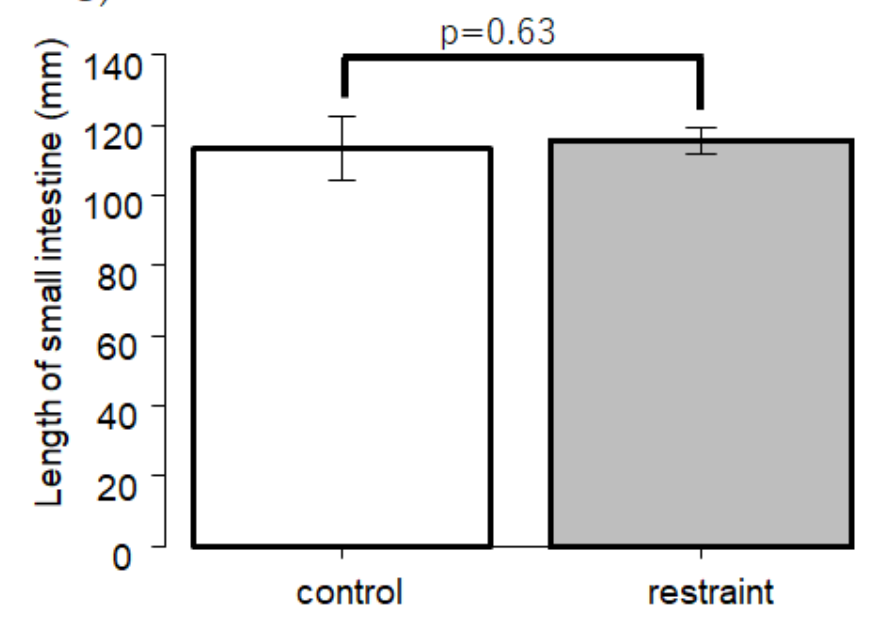

B)

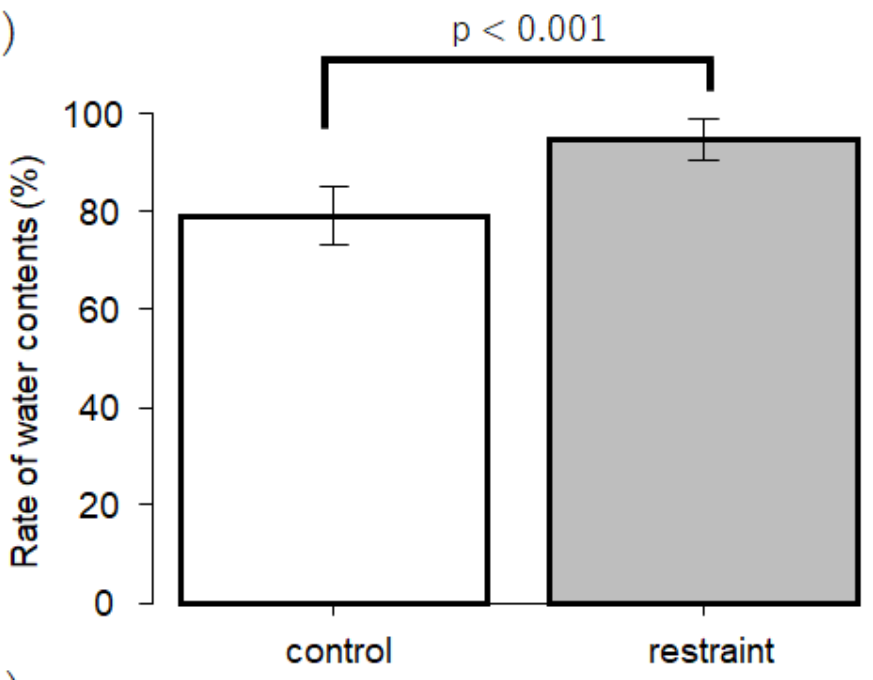

D)

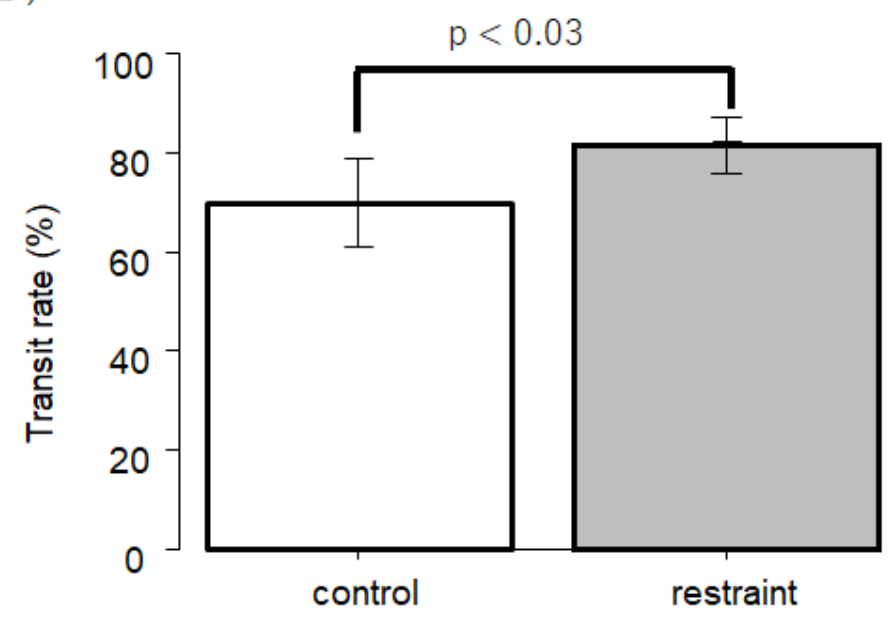

Figure 1

A: The number of fecal pellet outputs during 1 hour of isolation was counted. The restraint $(n=12)$ and control $(n=12)$ groups were compared. B: Comparison of the rate of fecal water content in the restraint $(n=6)$ and control $(n=6)$ groups. The first fecal pellet collected during isolation and after isolation were compared ((fecal weight before drying - fecal weight after drying) / fecal weight before drying (\%)). C: The length of the small intestine was compared between the restraint $(n=9)$ and control $(n=16)$ groups. $D$ : The transit rate of the small intestine was compared between the restraint $(n=9)$ and control $(n=16)$ groups. The small intestinal transit rate was calculated as the length of the small intestine containing the marker/total small intestinal length $\times 100 \%$. 
A)

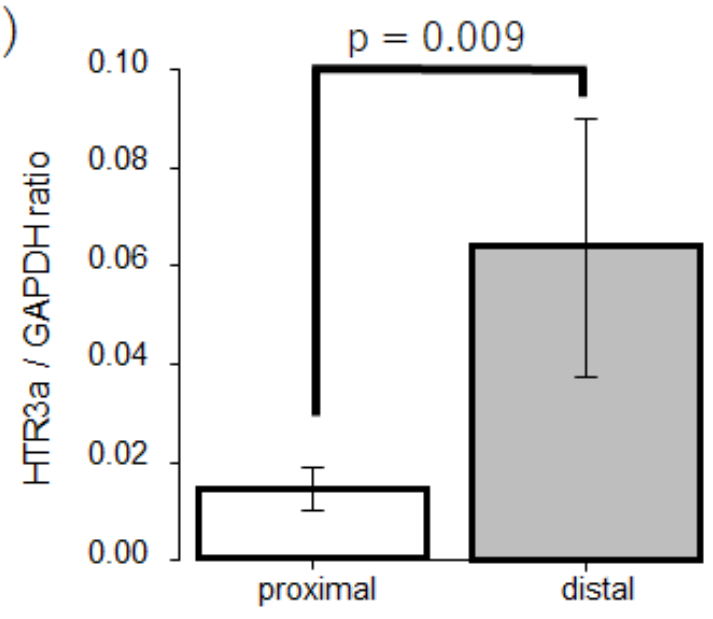

C)

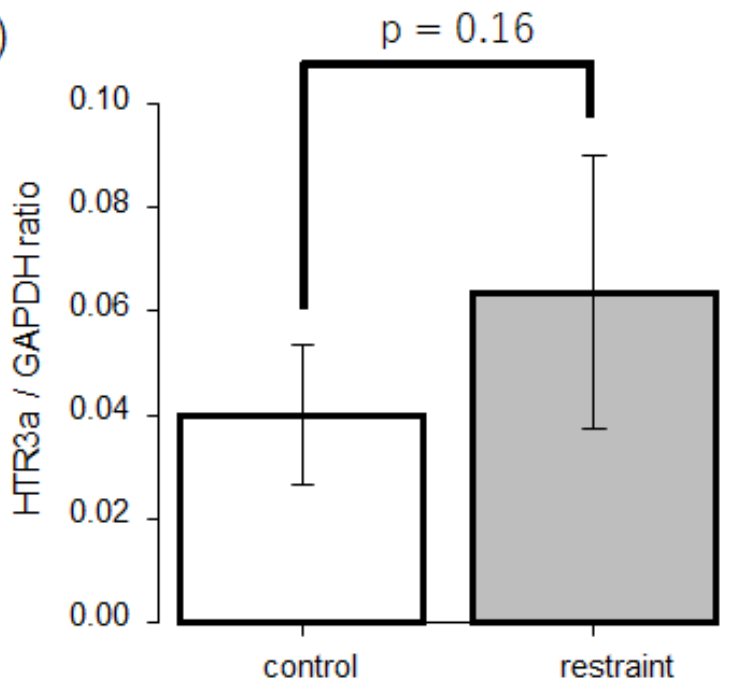

B)

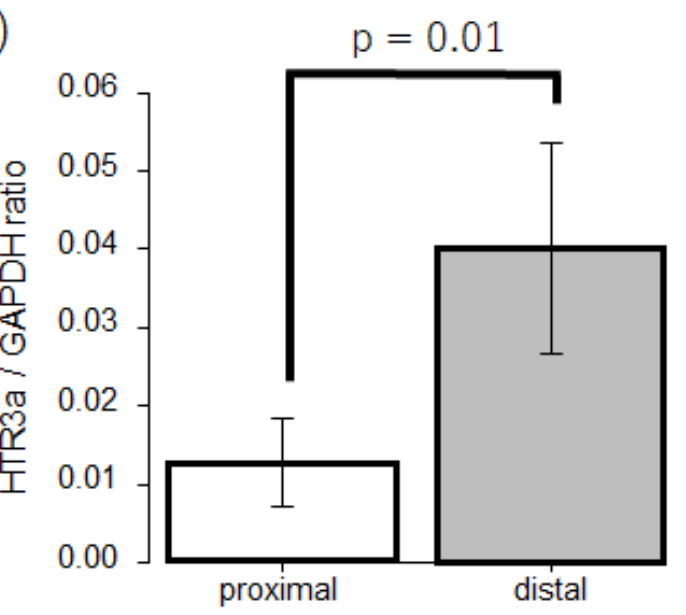

\section{Figure 2}

A: The expression of 5-Hydroxytryptamine Receptor 3A (5-HTR3a) in the proximal and distal small intestine was compared in the restraint group. B: The expression of 5-HTR3a in the proximal and distal small intestine was compared in the control group. C: The expression of 5-HTR3a was compared between the restraint and control groups only in the distal small intestine. 
A) Small intestine

Control

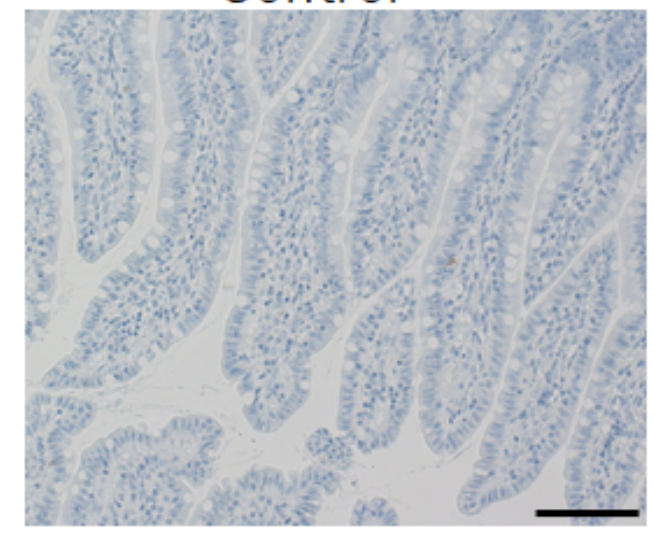

B) Colon
Control

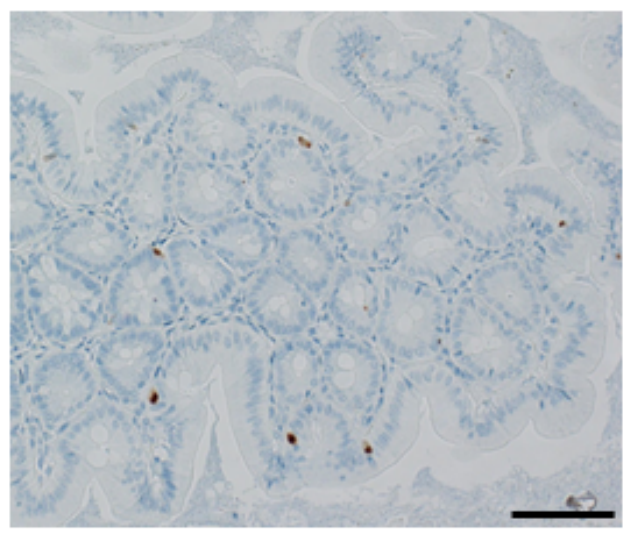

Restrain

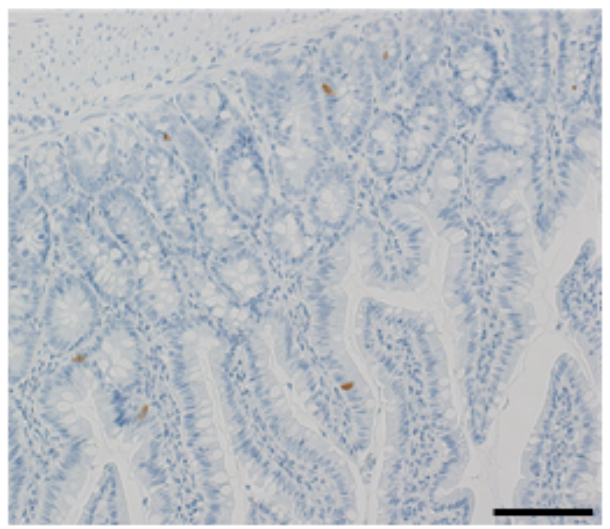

Restraint

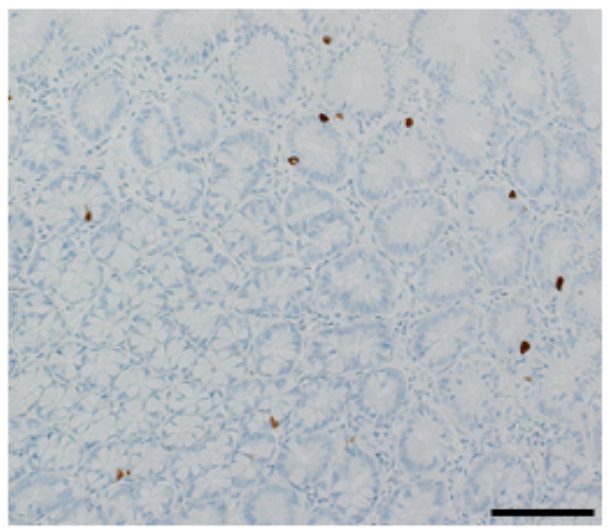

Figure 3

Enterochromaffin cell (EC cell) expression tended to increase in the restraint group compared to the control group in both the small intestine and colon. Bar $=100 \mu \mathrm{m}$. A: Photographs of the small intestine tissue. The positive cells (EC cells) were detected. The number of positive cells between the restraint and control groups was compared. B: Photographs of the colon tissue. The number of positive cells between the restraint and control groups was compared. 
A)

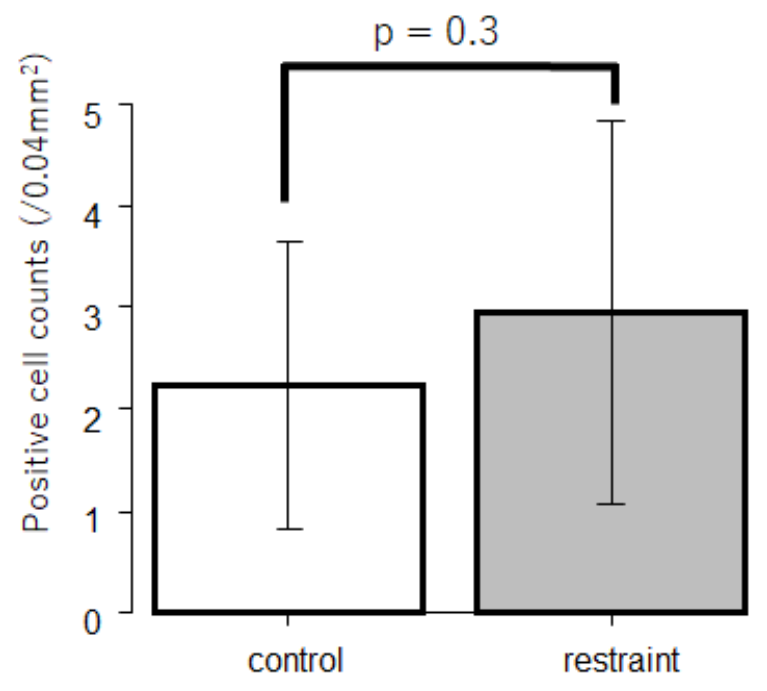

B)

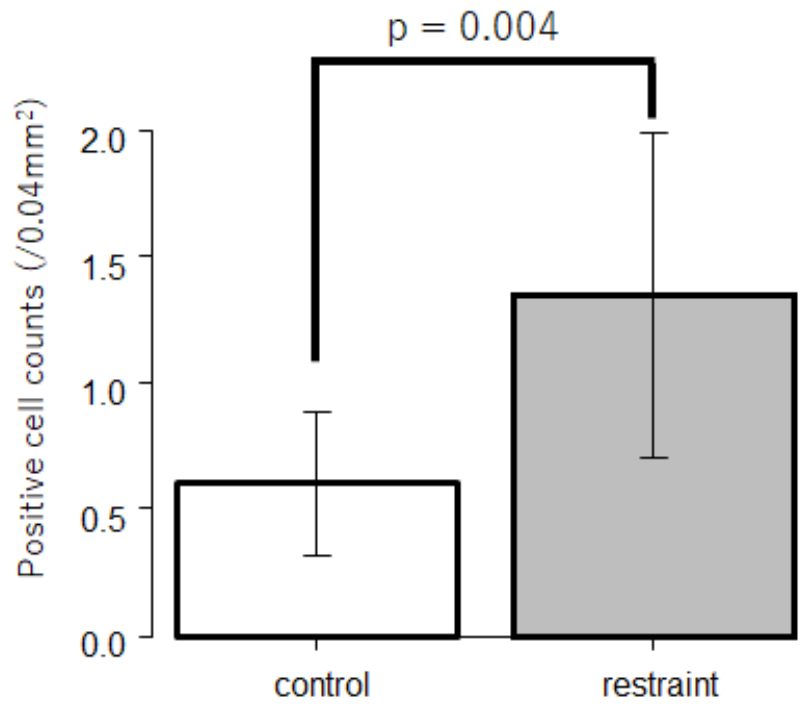

\section{Figure 4}

Comparison of the number of positive cells in the restraint $(n=6)$ and control $(n=6)$ groups and expression of $5-\mathrm{HT}$ in the small intestine and colon. $5-\mathrm{HT}$ is secreted from EC cells. EC cells that were stained and positive were counted. The number of EC cells in the small intestine increased in the restraint group, but not in the colon. A: The counted positive cells in the colon were compared between the restraint and control groups. B: The counted positive cells in the small intestine were compared between the restraint and control groups.

A)

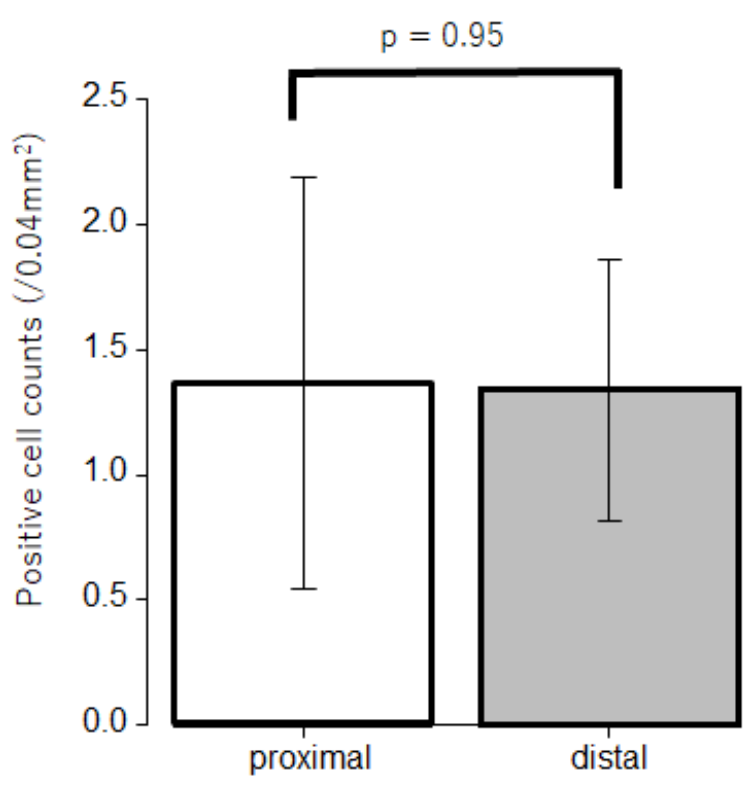

B)

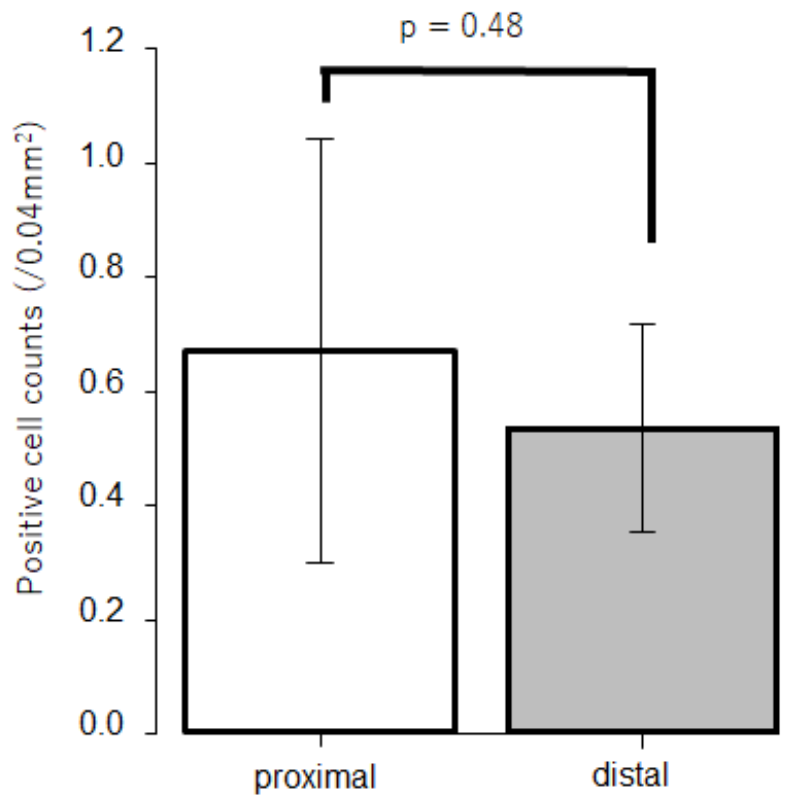

Figure 5 
A: Comparison of enterochromaffin cell (EC cell) expression between the proximal small intestine and the distal small intestine in the restraint group. B: Comparison of EC cell expression between the proximal small intestine and the distal small intestine in the control group.

A)

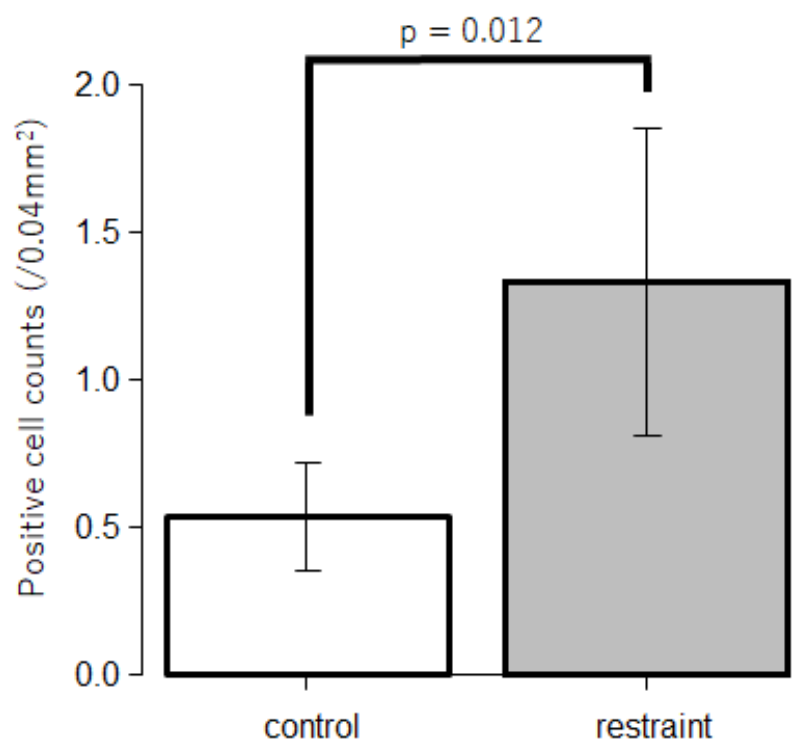

B)

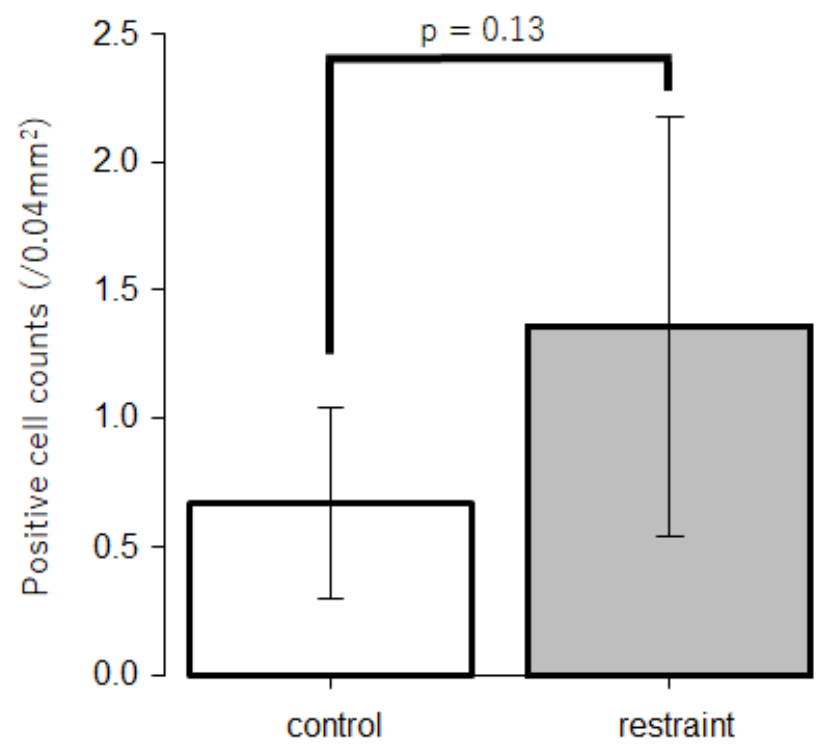

Figure 6

A: The number of Enterochromaffin cells (EC cells) in the distal small intestine was compared between the restraint and the control groups. B: The number of EC cells in the proximal small intestine was compared between the restraint and control groups. 
A) Small intestine

Control

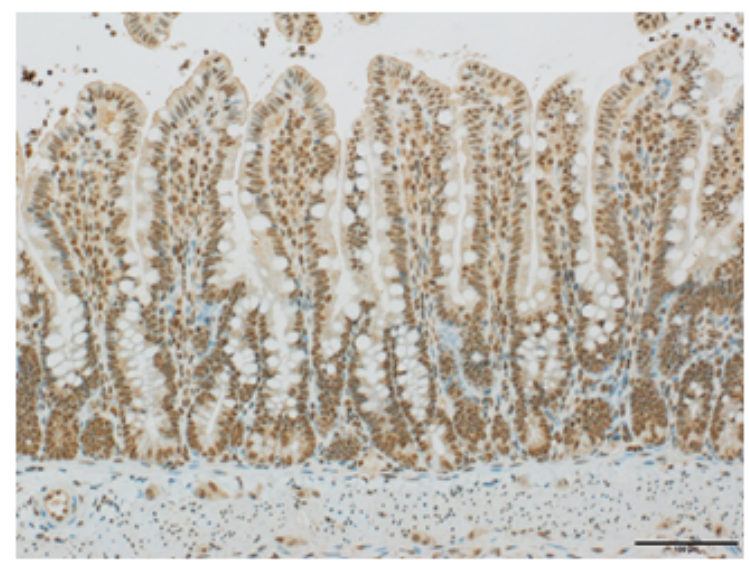

B) Colon

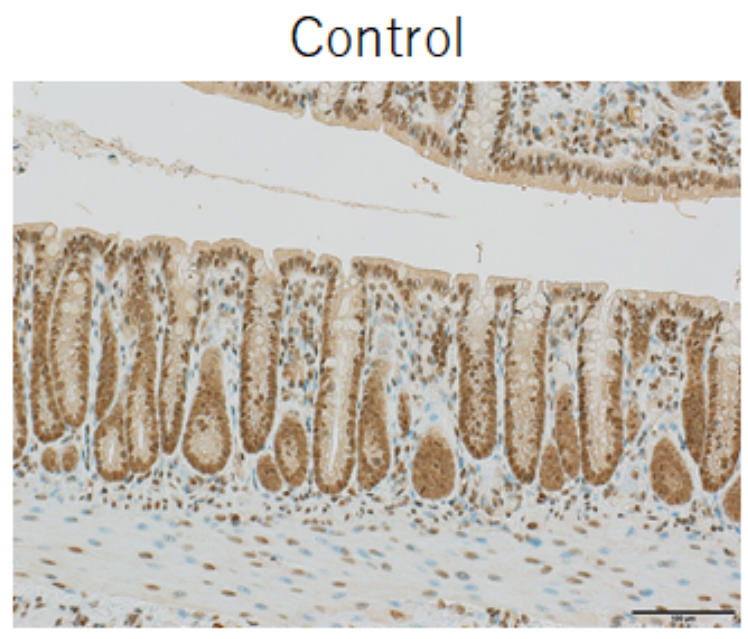

Restraint

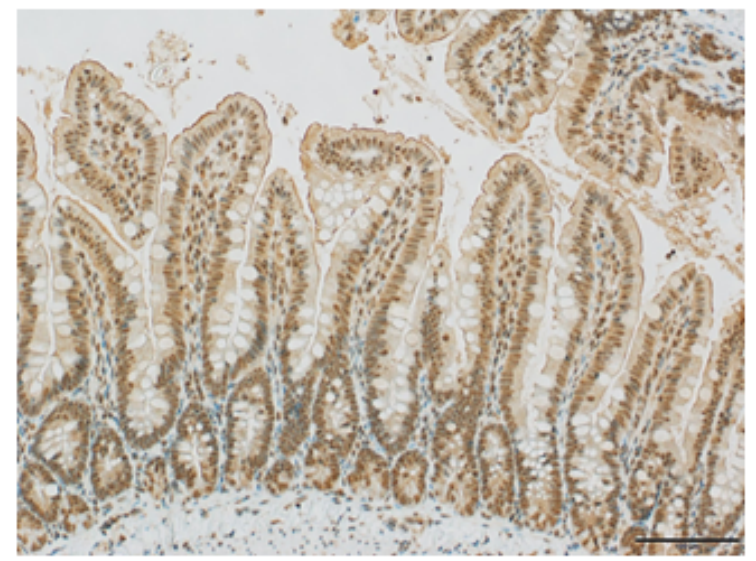

\section{Restraint}

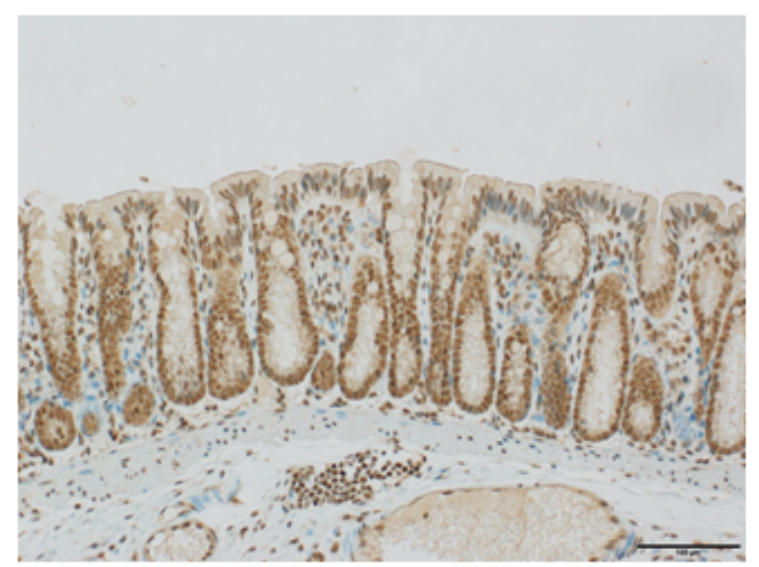

\section{Figure 7}

The expression of 5-Hydroxytryptamine Receptor 3A (5-HTR3a) in the small intestine and colon. Bar = $100 \mu \mathrm{m}$. A: Photographs of the small intestine tissue. The expression of 5-HTR3a between the restraint and control groups was compared. B: Photographs of the colon tissue. The expression of 5-HTR3a between the restraint and control groups was compared. 